\title{
CARACTERIZACIÓN LEUCOCITARIA DEL PEZ AMAZÓNICO Pterophyllum scalare (Lichtenstein, 1823) (PERCIFORMES: CICHLIDAE) DE PERÚ
}

\author{
José Iannacone $e^{1}$ \\ Cinthya Bello ${ }^{2}$ \\ Nancy Hernández ${ }^{2}$ \\ María Díaz ${ }^{2}$
}

\begin{abstract}
RESUMEN
La Ictiohematología es una herramienta de uso común con fines clínicos en peces de importancia económica, con un menor aporte en la comprensión de la fisiología del organismo en relación con su medio. En este contexto y frente a la escasa información publicada en el Perú de aspectos hematológicos de osteictios, se propuso caracterizar los leucocitos del pez angel Pterophyllum scalare (Lichtenstein, 1823) y determinar los valores de referencia cuantitativos. Este pez amazónico exportado como ornamental fue obtenido de un acuario de Lima-Perú. La extracción de la sangre, se llevó a cabo por el método de punción cardiaca, realizándose luego extensiones teñidas con Giemsa y con Wright, y se procedió a realizar el recuento leucocitario. Se relacionaron los parámetros biométricos [longitud estándar (mm), longitud total (mm) y peso (g)] con su caracterización leucocitaria. Los leucocitos, en general, presentaron características similares a las descritas para otros peces. Cuantitativamente, se encontró la siguiente secuencia: linfocitos $(61,6 \%)>$ monocitos $(30,1 \%)>$ neutrófilos $(4,0 \%)>$ eosinófilos (2,1\%)> basófilos (1,8\%). Los parámetros hematológicos obtenidos son un aporte al conocimiento de esta especie y servirán como base comparativa en futuras aplicaciones.
\end{abstract}

Palabras claves: Amazonía, leucocitos, parámetros hematológicos, Pterophyllum scalare.

\section{SUMMARY}

Ichthyohematology is a tool of a common employ for clinical aim in economic important fish, with a used less to knowledge of organism physiology in relation to its environment. In this context and face to scanty information published in Peru related to hematological aspects of bony fishes, characterization of leukocytes of angelfish Pterophyllum scalare (Lichtenstein, 1823) and determination of reference quantitative values was proposed. This Amazonian fish exported as ornamental was obtained from an aquarium of Lima, Peru. Blood extraction, was performed by cardiac puncture method, and then slides were stained with Giemsa and with Wright, and was proceed a total leukocyte count. Biometric parameters [standard length $(\mathrm{mm})$, total length (mm) and weight (g)] with its total leukocyte characterization were related. Leukocytes, in general, showed characteristics similar to description of other fish. Quantitatively, was found the following sequence: lymphocytes $(61.6 \%)>$ monocytes $(30.1 \%)>$ neutrophils (4.0\%)> eosinophils $(2.1 \%)>$ basophils $(1.8 \%)$. The hematological parameters obtained are important for the specie knowledge and will be useful as a comparative base for future studies.

Key words: Amazonian, leukocytes, hematological parameters, Pterophyllum scalare.

\footnotetext{
${ }^{1}$ Laboratorio de Invertebrados. Facultad de Ciencias Biológicas. Universidad Ricardo Palma. e-mail:jiannacone@mail.urp.edu.pe

2 Laboratorio de Ecofisiología Animal. Facultad de Ciencias Naturales y Matemática. Universidad Nacional Federico Villarreal.
} 


\section{INTRODUCCIÓN}

La Ictiohematología puede ser definida, en términos generales, como una disciplina que estudia la sangre de los peces; sin embargo, en términos prácticos esta especialidad estudia las células sanguíneas morfológica, bioquímica y funcionalmente como también los órganos hematopoyéticos, las enfermedades relacionadas con ellos y cualquier fenómeno o patología que relacione las células y/o sus órganos productores. De este modo, las variaciones de los parámetros hematológicos como hematocrito, leucocitos, recuentos celulares y concentración de hemoglobina pueden, entre otros, ser utilizados como indicadores de contaminación o estrés por parasitismo (Örün et al., 2003; Valenzuela et al., 2003; Cancino \& Santos de Aráoz, 2004; Aviléz et al., 2006; Franca et al., 2006; PereiraMaduenho \& Martinez, 2006). A su vez, los parámetros hematológicos están siendo utilizados como indicadores fisiológicos de disfunción orgánica por estrés (Örün \& Erdemil, 2002; Valenzuela et al., 2002). Así, por ejemplo, la tensión de oxígeno tiene implicancias directas en la regulación de la eritropoyesis de modo que la hipoxia produce, como respuesta aguda, la liberación de eritrocitos por contracción esplénica, causando aumento de policromatófilos (eritrocitos inmaduros) en circulación (Valenzuela et al, 2002); por otra parte, el estrés crónico produce leucopenia y cambios en la fórmula leucocitaria como linfopenia, monocitopenia y neutrofilia (Ueda et al., 2001).

La necesidad de establecer patrones hematológicos en peces surge de su utilidad como referencia para el diagnóstico de cuadros patológicos. Aunque la composición sanguínea de los peces esta determinada genéticamente, también se encuentra bajo la influencia del ambiente en el que habitan los organismos (Cancino \& Santos de Aráoz, 2004). Los estudios sobre la hematología de los peces han permitido comprobar experimentalmente que variaciones en las condiciones ambientales como temperatura, salinidad y oxigeno causan modificaciones fisiológicas en los niveles de algunos parámetros sanguíneos (Landman et al., 2005). En adición, malnutrición, edad, tamaño del pez, diferencias estacionales, pueden ocasionar variaciones en los parámetros hematológicos (Veiga et al., 2000; Örün et al., 2003; Ranzani-Paiva et al., 2003).

Las variaciones de los parámetros hematológicos como hematocrito, leucocito, recuentos celulares y concentración de hemoglobina pueden, entre otros, ser utilizados como indicadores de contaminación; así por ejemplo el estrés crónico produce leucopenia y cambios en la fórmula leucocitaria como linfopenia, monocitopenia y neutrofilia (Villalobos, 2002).

El pez ángel Pterophyllum scalare (Lichtenstein, 1823) presenta importancia como pez ornamental amazónico para exportación. Esta especie presenta un cuerpo alto y comprimido en forma romboide con la boca sobresalida. La talla que alcanza en cautiverio es de $15 \mathrm{~cm}$ de longitud y $25 \mathrm{~cm}$ de alto. $P$. scalare requiere aguas ligeramente ácidas (Acuanovel, 2006). La temperatura más adecuada se encuentra entre los $28^{\circ} \mathrm{C}$ y $30^{\circ} \mathrm{C}$. Esta especie presenta dimorfismo sexual, el cual es casi inexistente fuera de la época de cría, aunque se puede apreciar que el macho tiene un perfil corporal más redondeado que la hembra y una frente más abultada. Se han realizado varias investigaciones sobre el comportamiento y el parasitismo en el pez ángel bajo condiciones de laboratorio (Yamamoto et al., 1999; Gómez-La Plaza \& Morgan, 2003, 2005; Thilakaratue et al., 2003; Cacho et al., 2006).

Sobre $P$. scalare se ha desarrollado un creciente interés por utilizarlo como bioindicador de contaminación química en el Perú. Sin embargo, el conocimiento hematológico de peces ornamentales amazónicos en el Perú es muy escaso, lo cual crea la necesidad de aumentar el conocimiento de esta especie, obteniendo datos que puedan ser utilizados en su manutención tanto en su ambiente natural como en cautiverio y, además, servir como referencia para futuras investigaciones experimentales en el campo de la hematología comparada y otras disciplinas. El objetivo de este trabajo fue establecer el recuento leucocitario del pez angel $P$. 
scalare, y determinar su valores de referencia cuantitativos.

\section{MATERIAL Y MÉTODOS}

Dieciséis ejemplares del pez amazónico ornamental $P$. scalare se adquirieron del acuario «Cleo» en el distrito de Lince, Lima-Perú en el mes de Junio del 2006. Luego se procedió a su transporte al laboratorio de Ecofisiología Animal de la Facultad de Ciencias Naturales y Matemática de la Univerdad Nacional Federico Villarreal. La temperatura fluctuó entre 19 a $24^{\circ} \mathrm{C}$. Los peces fueron colocados en agua de grifo declorinada con las siguientes características fisicoquímicas: $\mathrm{pH}=7,2$; Conductividad eléctrica $=0,86 \mathrm{dS} \mathrm{m}^{-1}$; Calcio $=123,6 \mathrm{mg} \mathrm{L}^{-1}$; Magnesio $=15,3 \mathrm{mg} \mathrm{L}^{-1}$; Potasio $=3,90 \mathrm{mg}$ $\mathrm{L}^{-1}$; Sodio $=4,70 \mathrm{mg} \mathrm{L}^{-1}$; Nitratos $=3,10$ $\mathrm{mg} \mathrm{L}^{-1}$; Bicarbonatos $=216,5 \mathrm{mg} \mathrm{L}^{-1}$; Sulfatos $=110,4 \mathrm{mg} \mathrm{L}^{-1}$; Cloruros $=85,1$ $\mathrm{mg} \mathrm{L}^{-1}$; Boro $=0,5 \mathrm{mg} \mathrm{L}^{-1}$; Dureza cálcica $=362 \mathrm{mg} \mathrm{L}^{-1}$.

A cada ejemplar, se determinó el sexo, se registró la longitud total (Lt en $\mathrm{mm}$ ), longitud estándar (Ls en mm) y el peso corporal (P en g). Posteriormente, se les anestesió con Lidocaína al 2\% (Xilocaina ${ }^{\circledR}$ ), y después de 5 min se procedió a tomar muestras de sangre mediante el método de punción cardiaca. Se tomaron muestras de sangre para realizar los dos frotis sanguíneos. Posteriormente se realizaron los estudios leucocitarios. A cada ejemplar de $P$. scalare se le extrajo una gota de sangre confeccionando dos extendidos sobre portaobjetos. Una lámina fue coloreada con Giemsa y la otra con Wright. Los diferentes leucocitos fueron observados al microscopio óptico con un aumento de 1000x. Se realizó el recuento leucocitario de un total de 100 células (Örün \& Erdemil, 2002). Finalmente se obtuvieron 15 láminas coloreadas con Giemsa y 13 con Wright.

Para los valores del recuento leucocitario obtenidos y para los parámetros biométricos se realizó el cálculo de su media y de su desviación estándar. Además se empleó el coeficiente de correlación lineal de Pearson (r), entre los parámetros biométricos y el número de leucocitos caracterizados. Se comparó si el número de los diferentes tipos de leucocitos era diferente entre los machos y las hembras empleando la prueba de $t$ de student. En adición, se usó la prueba de $t$ de student para datos pareados para comparar si existen diferencias entre el recuento de leucocitario entre la coloración Giemsa y Wright. Se empleó el paquete estadístico SPSS, versión 12,00 para Windows 98 para el cálculo de los estadísticos descriptivos e inferenciales.

\section{RESULTADOS}

En el frotis sanguíneo realizado con los colorantes Giemsa y Wright para el recuento leucocitario de $P$. scalare se identificaron cinco tipos celulares (Tabla 1). A continuación se describen algunas características observadas en las cinco células leucocitarias:

Linfocitos: células redondeadas-esféricas, de forma irregular cuyo núcleo ocupa gran parte del citoplasma. Presentan cromatina condensada en grumos. El citoplasma es escaso, con algunas prolongaciones e intensamente basófilo. Se observaron linfocitos grandes y pequeños.

Monocitos: células grandes y esféricas con expansiones citoplasmáticas. Gran núcleo excéntrico con cromatina laxa y de citoplasma basófilo.

Neutrófilo: corresponden a granulocitos polimorfonucleares (PMN), generalmente con núcleo excéntrico, y lobulado. Cromatina condensada en grumos y citoplasma con finas granulaciones, con cuerpos basófilos.

Eosinófilo: células redondas pequeñas de núcleo excéntrico lobulado y con gránulos eosinófilos esféricos citoplasmáticos en bastón recto o curvo. Cromatina laxa.

Basófilo: células abastonadas con citoplasma basófilo.

Cuantitativamente, se encontró la siguiente secuencia promedio: linfocitos $(61,6 \%)>$ monocitos $(30,1 \%)>$ neutrófilos $(4,0 \%)>$ eosinófilos (2,1\%)> basófilos (1,8\%) (Tabla 1). Tampoco se observó que los recuentos porcentuales de células leucocitarias (linfocitos, monocitos, neutrófilos, eosinófilos y basófilos) fueran diferentes según las técnicas de coloración empleadas (Giemsa y Wright) (Tabla 1). 
Para la mayoría de los casos no se encontraron correlaciones significativas entre la Lt, Ls y el P con cada uno de los parámetros del recuento leucocitario $(\mathrm{P}>$ $0,05)$. Sin embargo, se encontró correlación positiva entre la Lt y el recuento de linfocitos en Wright ( $r=0,67 ; p=0,02)$. P se encontró relacionado positivamente con el recuento de basófilos en Wright $(r=0,86$; $p=0,001)$. El aumento de linfocitos en Giemsa estuvo negativamente relacionado con el número de monocitos en Giemsa ( $\mathrm{r}=$ -0,84; $\mathrm{p}=0,00$ ). El mismo patrón se observó en Wright $(r=-0,71 ; p=0,01)$. El porcentaje de basófilos en Giemsa presentó una correlación positiva con el número neutrófilos en Giemsa $(r=0,84 ; p=0,00)$.

No existieron diferencias entre los peces machos $(n=9)$ y hembras $(n=7)$ con relación a ninguno de los tres parámetros biométricos y para el porcentaje de los diferentes tipos de leucocitos (Tabla 2). La única excepción fue observar un mayor número de monocitos en la coloración Giemsa en los machos (Tabla 2).

\section{DISCUSIÓN}

Las células sanguíneas de los frotis que analizamos en las lecturas fueron similares morfológicamente a las descritas para Shroedenchthys chilensis (Guichenot, 1848) (Filho et al., 1992; Valenzuela et al., 2003), Salminus maxillosus Valenciennes, 1840 (Veiga et al., 2000; Ranzani-Paiva et al., 2003) y Chaetodipterus faber (Broussonet, 1782) (Bastardo \& Barberán, 2004). En el recuento se observó un predominio de de los linfocitos, lo cual es concordante con lo encontrado en otros peces teleósteos como Pimelodus maculatus Lacèpéde, 1803, Synbranchus marmoratus (Bloch, 1795), Mugil platanus Günther 1880 y Oncorhynchus mykiss (Walbaum, 1792) (Örün \& Erdemil, 2002; Ranzani-Paiva et al., 2003; Valenzuela et al., 2003). Los trombocitos no han sido identificados en el presente estudio, posiblemente debido a que frecuentemente son confundidos con los linfocitos y considerados un solo grupo al ser difícil su identificación (Ueda et al., 2001). Estos últimos autores recomiendan el empleo de la citoquímica como una herramienta para la identificación de los trombocitos y linfocitos. Tavares-Dias (2006) señala procedimientos citoquímicos para la diferenciación de los basófilos.

Los valores del recuento leucocitario entre individuos de la misma especie y entre otras especies son variables. Entre los factores que alteran estos parámetros se citan la dieta, el linaje, la edad, el sexo, la estación del año, la cantidad de oxígeno disuelto, la madurez sexual y métodos de muestreo empleado (Cancino \& Santos de Aráoz, 2004). En el pez ángel, se encontró un mayor recuento porcentual de monocitos en machos que hembras. Örüm \& Erdemil (2002) encontraron para el pez Capoeta trutta (Heckel, 1843) (Cyprinidae) un mayor número de monocitos en las hembras que en los machos. En otros tres peces ciprinidos Alburnoides bipunctatus (Bloch, 1782), Chalcalburnus mossulensis (Heckel, 1843) y Cyprinion macrostomus (Heckel, 1843) se observó un mayor número de monocitos en hembras que en machos, atribuyéndosele al incremento de la actividad reproductiva y desarrollo gonadal, así como a mecanismos de defensa del pez por infecciones parasitarias (Örün et al., 2003; Ranzani-Paiva et al., 2003). Örüm \& Erdemil (2002) encontraron que el aumento de la longitud del pez C. trutta está relacionada con una disminución del número de linfocitos. En el presente trabajo en peces ángel de tamaño promedio pequeño, se encontró un modelo opuesto, es decir un aumento de los linfocitos con el aumento de la Lt. En cambio, RanzaniPaiva et al. (2003) encontró que la longitud total de S. maxillosus no influenció las variables hematológicas.

Algunos autores recomiendan no anestesiar a los peces antes de la toma de las muestras de sangre para recuento leucocitario pues pudiera afectar los resultados obtenidos (Örün \& Erdemil, 2002). Por ende, este procedimiento realizado pudiera influenciar los resultados obtenidos para los parámetros de recuento de leucocitos totales en el pez ángel.

Los resultados del presente estudio pudieran ser útiles en obtener valores estándar para parámetros hematológicos de P. scalare. 


\section{CONCLUSIONES}

En $P$. scalare, los leucocitos, en general, presentaron características similares a las descritas para otros peces. Cuantitativamente, se encontró la siguiente secuencia: linfocitos $(61,6 \%)>$ monocitos $(30,1 \%)>$ neutrófilos $(4,0 \%)>$ eosinófilos $(2,1 \%)>$ basófilos $(1,8 \%)$. Mayormente no se observó variación de los parámetros leucocitarios con el sexo y con el crecimiento de los peces.

\section{LITERATURA CITADA}

AQUANOVEL. 2006. Revista de acuariofilia marina y acuarios de agua dulce. Disponible en: http:// www.aquanovel.com/ leído 15 de Julio del 2006.

AVILÉZ, I.M.; HORI, T.F.S.; HONORATO, C.A.; CUNHABASTOS, J.; CUNHA-BASTOS, V.L.F. y MORAES, G. 2006. Efeitos tóxicos do fenol em eritrócitos de Brycon cephalus, matrinxã (Pisces, Characidae). p. 119. IX Congresso Brasileiro de Ecotoxicologia. 03 a 06 de julho de 2006, São Pedro, SP, Brasil.

BASTARDO A. y BARBERÁN, R. 2004. Parámetros hematológicos de la paragua, Chaetodipterus faber (Broussonet) (Pices: Ephippidae), en condiciones de cultivo. Zootecnia Tropical. 22: 361-370.

CACHO, M.S.R.F.; CHELLAPPA, S. y YAMAMOTO, M.M. 2006. Reproductive success and female preference in the amazonian cichlid angel fish, Pterophyllum scalare (Lichtenstein, 1823). Neotropical Ichthyology. 4: 87-91.

CANCINO, F. y SANTOS DE ARÁOZ, V. 2004. Parámetros hematológicos de Astyanax abramis (Jenyns, 1842) (Characiformes, Characidae) del embalse Río Hondo, Santiago del Estero-Tucumán. Acta zoológica lilloana. 48: 81-89.

FILHO, W.; EBLE, G.J.; KASSNER, G.; CAPRARIO, F.X.; DAFRE, A.L. y OHIRA, M. 1992. Comparative hematology in marine fish. Comp.
Biochemical Physiology and Comparative Physiology. 102: 311321.

FRANCA, J.G.; RANZANI-PAIVA, M.J.T.; LOMBARDI, J.V.; SERIANI, R.; CARVALHO, S. \& ANDREGHETTO, F.M. 2006. Avaliação do efeito do selénio sobre a ação subletal do cloreto de mercúrio em tilapia, Oreochromis niloticus, através de análises hematológicas.p. 105. IX Congresso Brasileiro de Ecotoxicologia. 03 a 06 de julho de 2006, São Pedro, SP, Brasil.

GÓMEZ LAPLAZA, L.M. y MORGAN, F. 2003. The influence of social rank in the angelfish, Pterophyllum scalare, on locomotor and feeding activities in a novel environment. Laboratory Animal. 37: 108-120.

GÓMEZ LAPLAZA, L.M. y MORGAN, F. 2005. Time-place learning in the angelfish, Pterophyllum scalare. Behavior Processes. 70: 177-181.

LANDMAN, M.J.; VAN DEN HEUVEL, M.R.; FINLEY, M.; BAÑÓN, H.J. y LING, N. 2005. Combined effects of pulp and paper effluent, dehydroabietic acid, and hypoxia on swimming peroformance, metabolism, and hematology of rainbow trout. Ecotoxicology Environmental Safety. (En prensa).

ÖRÜN, I.; DÖRÜCÜ, M. y YAZIAK, H. 2003. Haematological parameters of three cyprinid fish species from Karakaya Dam Lake, Turkey. OnLine Journal of Biological Sciences. 3: 320-328.

ÖRÜM, I. y ERDEMIL, A. U. 2002. A study of blood parameters of Capoeta trutta (Heckel, 1843). OnLine Journal of Biological Sciences. 2: 508-511.

PEREIRA-MADUENHO, L. $\mathrm{y}$ MARTINEZ, C.B. R. 2006. Efeitos hematológicos, iónicos e metabólicos do inseticida dimilin para o peixe neotropical Prochilodus lineatus. p. 41. IX Congresso Brasileiro de Ecotoxicologia. 03 a 06 de julho de 2006, São Pedro, SP, Brasil.

RANZANI-PAIVA, M.J.T.; RODRIGUEZ, E.L.; VEIGA, M.L.; EIRAS, A.C. y CAMPOS, B.E.S. 2003. 
Differential leukocyte counts in «dorado» Salminus maxillosus Valenciennes, 1840, from the mogiguacu river, Pirassununga, SP. Brazilian Journal of Biology. 63: 517525.

TAVARES-DIAS. 2006. Cytochemical method for staining fish basophils. Journal of Fish Biology. 69: 312-317.

THILAKARATUE, I.D.; RAJAPAKSHA, G.; HEWAKOPARA, A.; RAJAPAKSE, R.P. y MAIZAL, A.C. 2003. Parasitic infection in freswater ornamental fish in Sri Lanka. Disease of Aquatic Organism. 54: 157-162.

UEDA, I.K.; EGAMI, M.I.; SASSO, W.S. y MATUSHIMA, E.R. 2001. Cytochemical aspects of the peripherical blood cells of Oreochromis (Tilapia) niloticus. (Linnaeus, 1758) (Cichlidae: Teleostei)- Part II. Brazilian Journal of Veterinary Researchin Animal Science. 38: 273-277.

VALENZUELA, A.; ALVEAL, K. y TARIFEÑO, E. 2002. Respuesta hematológica de truchas (Oncorhynchus mykiss Walbaum 1792) a estrés hipóxico agudo: serie roja. Gayana. 66: 255-261.
VALENZUELA, A. C., OYARZÚN, C. y SILVA, V. 2003. Células sanguíneas de Schroederichthys chilensis (Guichenot 1848) (Elasmobranchii, Scyliorhinidae): la serie Blanca. Gayana. 67: 130-136.

VEIGA, M.L.; EGAMI, M.I.; RANZANIPAIVA, M.J.T. y RODRIGUES, E.L. 2000. Aspectos morfológicos y citoquímicos de las células sanguíneas de Salminus maxillosus Valenciennes, 1840 (Characiformes, Characidae). Revista Chilena de Anatomía. 18: 245-250.

VILLALOBOS, O. 2002. Pez Ángel o Escalar (Pterophyllum scalare) ACUARAMA. No3. Disponible en: http://c.1asphost.com/aavforo/ ACUARAMA_N_3.pdf leído el 15 de Julio del 2006.

YAMAMOTO, M.E.; CHELLAPPA, S.; CACHO, M.S.R.F. $\mathrm{y}$ HUNTINGFORD. 1999. Mate guarding in an amazonian cichlid, Pterophyllum scalare. Journal of Fish Biology. 55: 888-891.

Tabla 1. Recuento leucocitario bajo coloración por Giemsa y Wright en P. scalare.

\begin{tabular}{llllll}
\hline & & Wright* & \multicolumn{3}{c}{ Giemsa* } \\
\hline Leucocitos & M total \% & M \% $\pm \mathbf{D E}$ & M \% $\pm \mathbf{D E}$ & t & P \\
\hline Linfocitos & 61,6 & $54,8 \pm 10,5$ & $58,6 \pm 12,8$ & 1,44 & 0,18 \\
Monolitos & 30,5 & $35,6 \pm 12,8$ & $31,1 \pm 13,5$ & 1,80 & 0,10 \\
Neutrofilos & 4,0 & $4,9 \pm 3,7$ & $4,3 \pm 5,0$ & 0,45 & 0,66 \\
Eosinofilos & 2,1 & $2,2 \pm 3,7$ & $1,2 \pm 1,3$ & 0,86 & 0,41 \\
Basofilos & 1,8 & $1,6 \pm 2,5$ & $2,6 \pm 4,0$ & 1,06 & 0,31 \\
\hline
\end{tabular}

$\mathrm{M}=$ Promedio. $\mathrm{DE}=$ Desviación estándar. $\mathrm{t}=\mathrm{t}$ de Student para muestras pareadas. $\mathrm{P}=$ Probabilidad. *= Coloración

Tabla 2. Parámetros leucocitarios y biométricos en machos y hembras de $P$. scalare.

\begin{tabular}{lllll}
\hline & Macho & Hembra & t & P \\
\hline Lt (mm) & $23,8 \pm 8,1$ & $20,4 \pm 3,5$ & 0,90 & 0,38 \\
Ls (mm) & $19,8 \pm 5,9$ & $16,4 \pm 4,3$ & 1,14 & 0,27 \\
P (g) & $0,42 \pm 0,49$ & $0,36 \pm 0,30$ & 0,21 & 0,83 \\
Linfocito (\%) G & $54,5 \pm 10,9$ & $68,8 \pm 14,4$ & 2,03 & 0,06 \\
Linfocito (\%) W & $61,0 \pm 17,2$ & $62,3 \pm 10,6$ & 0,12 & 0,90 \\
Monocito (\%) G & $38,5 \pm 12,7$ & $20,6 \pm 9,2$ & 2,71 & $0,02^{*}$ \\
Monocito (\%) W & $28,8 \pm 17,7$ & $28,0 \pm 7,8$ & 0,08 & 0,93 \\
Neotrófilo (\%) G & $3,3 \pm 3,1$ & $4,8 \pm 4,9$ & 0,65 & 0,53 \\
Neutrófilo (\%) W & $4,4 \pm 5,7$ & $3,0 \pm 1,7$ & 0,39 & 0,70 \\
Eosinófilo (\%) G & $1,2 \pm 2,3$ & $4,6 \pm 4,6$ & 1,74 & 0,11 \\
Eosinófilo (\%) W & $1,2 \pm 2,1$ & $2,6 \pm 1,1$ & 1,11 & 0,29 \\
Basófilo (\%) G & $1,1 \pm 1,8$ & $1,4 \pm 3,1$ & 0,20 & 0,84 \\
Basófilo (\%) W & $1,8 \pm 3,5$ & $4,0 \pm 5,3$ & 0,83 & 0,42 \\
\hline
\end{tabular}

$\mathrm{G}=$ Coloración Giemsa. $\mathrm{W}=$ Coloración Wright. $\mathrm{t}=\mathrm{t}$ de Student. $\mathrm{P}=$ Probabilidad * = Significativo. 